\title{
The Early Impact of COVID-19 on Cancer Education and Cancer Control
}

\author{
Clement K. Gwede ${ }^{1}$ \\ Published online: 11 January 2021 \\ (C) American Association for Cancer Education 2021
}

As the president of the American Association for Cancer Education, I would like to provide a brief reflection on the early effects of the coronavirus pandemic and coronavirus disease (COVID-19) on our core vision "achieving excellence in education to reduce the burden of cancer worldwide." The global coronavirus pandemic continues to rage worldwide, and its impact on cancer education and cancer control has spanned many aspects from cancelation, interruption, delay, or transition to virtual formats for (1) cancer education, training and mentoring activities at all levels of health career development, training and academic/professional learning, to (2) cancer treatment/care delivery to patients with active cancer, and (3) delays/adjustments in receipt of guidelinerecommended prevention, screening and early detection modalities among populations in need [1-3]. Across these three pillars of cancer education and cancer control, some activities have resumed in-person or in mixed hybrid approaches (virtual and in-person) or virtual only (e.g., videoconference education, telemedicine) or other contactless approaches (e.g., drive-through). Inevitably, there is growing new public optimism regarding vaccinations with the recent or imminent approval of at least two COVID-19 vaccines that appear to have high efficacy in preventing severe COVID-19 disease. The recent approval and first-ever vaccine administrations (to selected priority populations) in the United Kingdom, Canada and the United States and many other countries, is fueling heightened professional and public excitement.

This editorial focuses primarily on the immediate and obvious question of the impact of COVID-19 on cancer care delivery including prevention, screening, and early detection and treatment; areas that potentially threaten to wipe away the growing gains in cancer survival and consequently widening the disparate burden of cancer among medically and socially

Clement K. Gwede

Clement.Gwede@moffitt.org

1 Moffitt Cancer Center and University of South Florida, 12902 Magnolia Dr., FOW-EDU, Tampa, FL 33612, USA marginalized groups. To date, it is evident that the coronavirus pandemic has placed uniquely extraordinary constraints on cancer education activities in the areas of prevention, community education, and care delivery across the downstream spectrum of cancer control. As part of the 2020 International Cancer Education Conference, the early global impact of the pandemic was broached in key plenary presentations (October 12-16) as summarized by the list of titles below [4]. Six keynote scientific sessions addressed topics related to the theme of the conference "Using Cancer Education to Address Social Determinants of Health" $[4,5]$. These plenary presentations enriched our understanding of disparities, eLearning/education/training, social determinants of health, and the early global impact of the COVID-19 pandemic [4]. Specifically,

- Dr. Webb Hooper from the National Institute of Minority Health and Health Disparities (NIMHD) delivered the first plenary session, "Cancer Education in the Age of COVID19: Disparities, Pandemics, and Infodemics."

- The next day, Dr. Alison Lin, of the National Cancer Institute (NCI) discussed "Diversity in Cancer TrainingTransforming Challenges into Opportunities,"

- On the same day, Dr. Robin Vanderpool, of NCI presented on "Using Cancer Communication to Address Social Determinants of Health,"

- On the last day, Dr. Chris Lathan, Dana-Farber Cancer Institute, addressed "Cancer Disparities and Treatment Equity: Removing Barriers to Cancer Care in our Community";

- Dr. Stephen Thomas, University of Maryland Center for Health Equity, closed the conference with a pointed talk on impact of COVID-19 on communities of color, "The Colors of COVID-19: Confronting Health Disparities During a Global Pandemic," and

- Keeping a global perspective, the European Association for Cancer Education (EACE) plenary lecture on Thursday, provided by Dr. Charles Kelley addressed, "Teaching and eLearning in Cancer in a Time of COVID." 
The adverse impact of the COVID-19 pandemic on cancer prevention among the screening-eligible public and on cancer treatment/care delivery to patients with active cancer is emerging [1]. For example, a recent survey suggested $64 \%$ of people reported a scheduled mammogram, colonoscopy, skin check, and PAP/HPV test during the pandemic was delayed or canceled due to COVID-19, and $63 \%$ reported concern about being behind on their cancer screening [1]. Regarding patients with cancer, the survey [1] also reported that $58 \%$ of people with active cancer experienced disruptions in their daily lives; black patients $(61 \%)$ were more likely to report major disruptions compared to white patients $(47 \%)$, due to concerns over the risk of contracting the coronavirus.

Furthermore, missed screenings and delayed diagnoses due to the pandemic are projected to lead to more cancer deaths over the next 10 years. Specifically, NCI data estimated almost 10,000 additional US deaths from breast and colorectal cancers due to the COVID-19 pandemic between 2020 and 2030 [3]. Similar delays in cancer diagnosis were projected to cause as many as 3621 additional deaths within 5 years in the United Kingdom, based on a review of patients with just four tumor types [6]. As such, these early projections suggest a grim toll of the pandemic on important aspects of cancer prevention and cancer care delivery on patients and health systems. The full burden of the pandemic on cancer prevention and control may be unforeseeable at this time. These conditions have major implications not only for cancer patients and the general public needing cancer screenings but also for health system capacity such as cancer education and training (of the future workforce). As the long-term impact remains evolving and yet to be fully realized, proactive steps and considerations are needed right now.

Taken together, the broad global challenges imposed by the COVID-19 pandemic and the continuing racial/social injustices $[5,7]$ have further reinforced the importance of addressing social determinants in cancer education and continued support of efforts to end racial discrimination and social inequities. As a grim 2020 comes to an end, and the world welcomes the exciting promises of 2021, we must remain vigilant:

1. Institutions and individuals must maintain the allimportant COVID-19 risk mitigation measures (such as mask-wearing, hand washing, and social/physical distancing as indicated) for the foreseeable future, perhaps until mid-to-late 2021.

2. Individuals must make concrete plans for receiving appropriate COVID-19 vaccination as the vaccines become more widely available for all in 2021;

3. Cancer education professionals and health systems must continue to transition cancer education and research for changing realities and for a "post-COVID-19 normalcy," making sure everyone benefits. Is it the case of the glass "half-full" or "half-empty"? Will our activities fully return to pre-COVID-19 conditions, or are we looking at mixedapproaches going forward to reach everyone?

It is evident that the COVID-19 pandemic has already had an enormous impact on healthcare delivery in the USA and beyond, including an impact on cancer care delivery and prevention, screening, and early detection [2, 6, 8, 9]. As most healthcare systems have had to defer or modify cancer education and screening activities over the course of the pandemic, there has emerged heightened concern about future increases in incidence and outcomes in the foreseeable future. With improved COVID-19 safety procedures and continued risk mitigation measures allowing for some return to "normalcy" in many healthcare facilities, it remains unclear which groups of patients and the general public are effectively maintaining their recommended screening and early detection per guidelines. The general public and individuals at high risk for cancer must be supported to effectively engage in the safe resumption of guideline-based cancer education and screening to reduce both the overall burden of cancer and systemic, regrettable disparities affecting underserved groups to ensure health equity $[1-3,6,8,9]$.

\section{References}

1. Blanke CD (2020) COVID's impact on cancer prevention and care. Available from: https://www.swog.org/news-events/news/2020/10/ 23/covids-impact-cancer-prevention-and-care. Published October 23, 2020. Accessed 10 Dec 2020

2. DuBois RN (2020) COVID-19, cancer care and prevention. Cancer Prev Res. https://doi.org/10.1158/1940-6207.CAPR-20-0468

3. Sharpless NE (2020) COVID-19 and cancer. Science 368(6497): 1290

4. Bishop MC (2020) International cancer education conference: using cancer education to address social determinants of health. J Cancer Educ 35:1049-1051

5. Gwede CK (2020) Bringing a spotlight to the influences of social determinants of health. J Cancer Educ 35:211-213

6. Maringe C, Spicer J, Morris M, Purushotham A, Nolte E, Sullivan R, Rachet B, Aggarwal A (2020) The impact of the COVID-19 pandemic on cancer deaths due to delays in diagnosis in England, UK: a national, population-based, modelling study. Lancet Oncol 21:10231034

7. Braithwaite R, Warren R (2020) The African American Petri Dish. J Health Care Poor Underserved 1-12

8. Carethers JM, Sengupta R, Blakey R, Ribas R, D'Souza G (2020) Disparities in cancer prevention in the COVID-19 era. Cancer Prev Res 13:893-896

9. Weaver CH (2020) Studies Evaluate Impact of COVID-19 on Cancer Care. Available from: http://news.cancerconnect.com > treatment-care > studies-eval... Published Aug 10th, 2020. Accessed 10 Dec 2020

Publisher's Note Springer Nature remains neutral with regard to jurisdictional claims in published maps and institutional affiliations. 